\title{
Clave taxonómica del Superorden Squalomorphi de Chile (Pisces: Elasmobranchii)
}

\author{
Taxonomic key of the Superorder Squalomorphi from Chile (Pisces: Elasmobranchii)
}

Sylvia Sáez ${ }^{1}$, Germán Pequeño ${ }^{1}$ y Julio Lamilla ${ }^{1}$

${ }^{1}$ Instituto de Zoología “Ernst F. Kilian”, Universidad Austral de Chile, Casilla 567, Valdivia, Chile. sylvia.saez@gmail.com

\begin{abstract}
A taxonomic key to identify the Chilean species of the shark Superorder Squalomorphi, grouped in 16 families is presented. The families are: Hexanchidae, Chlamydoselachidae, Rhincodontidae, Pseudocarchariidae, Lamnidae, Cetorhinidae, Alopiidae, Scyliorhinidae, Triakidae, Carcharhinidae, Sphyrnidae, Dalatiidae, Centrophoridae, Squalidae, Echinorhinidae and Squatinidae. For the 56 currently considered species, the geographic distribution is indicated.
\end{abstract}

Key words: Taxonomy, sharks, geographic distribution

Resumen.- Se presenta una clave taxonómica para reconocer las especies chilenas de los tiburones del Superorden Squalomorphi, agrupadas en 16 familias: Hexanchidae, Chlamydoselachidae, Rhincodontidae, Pseudocarchariidae, Lamnidae, Cetorhinidae, Alopiidae, Scyliorhinidae, Triakidae, Carcharhinidae, Sphyrnidae, Dalatiidae, Centrophoridae, Squalidae, Echinorhinidae y Squatinidae. Para cada una de las 56 especies consideradas se indica la distribución geográfica.

Palabras clave: Taxonomía, tiburones, distribución geográfica

\section{INTRODUCCIÓN}

El Superorden Squalomorphi constituye un grupo de peces relativamente numeroso en Chile (Bahamonde \& Pequeño 1975, Meléndez \& Meneses 1986, Pequeño 1989, 1997), el cual no ha estado ajeno a cambios taxonómicos.

Las principales actualizaciones se han presentado en el Orden Squaliformes (Compagno 1984a, 1984b, Compagno 1999). Especies pertenecientes a este orden se agrupaban en dos familias: Echinorhinidae y Squalidae (Meléndez \& Meneses 1986, Pequeño 1989, Lloris \& Rucabado 1991, Sielfeld \& Vargas 1992, Pequeño 1997), actualmente se agrupan en cuatro familias debido a la subdivisión de la familia Squalidae en Dalatiidae, Centrophoridae y Squalidae (Taniuchi \& Garrick 1986, Nelson 1994). De estas familias principalmente Dalatiidae y Squalidae han presentado cambios en su integración e.g., Centrophorus squamulosus (Gunther, 1877), especie que antes pertenecía a Squalidae luego cambió a Scymnodon squamulosus (Regan, 1906) y actualmente es válido como Zameus squamulosus (Gunther, 1877) (Taniuchi \& Garrick 1986, Meléndez 1991), que fue hallazgo para Chile en aguas del golfo de Ancud (Meléndez \& Céspedes 1996) y de la familia Squalidae pasa a Centrophoridae (Nelson 1994, Eschmeyer \& Fricke 2010). Scymnodon macracanthus (Regan, 1906) es válido como Centroscymnus macracanthus (Regan, 1906) (Compagno 1984a, 2001) y Squalus fernandinus Molina, 1782 fue excluida de Squalidae por considerarse sinónimo de Squalus acanthias Linnaeus, 1758, quedando la familia con sólo tres especies (Compagno 1984a, Lloris \& Rucabado 1991).

En la familia Carcharhinidae, existe confusión debido al estado incierto de dos de sus taxa descritos: Hypoprion isodus Philippi, 1887 e H. heterodus Philippi, 1887 y la familia quedó reducida a dos especies, Prionace glauca y Carcharhinus galapagensis (Garrick 1982, Pequeño 1989). En el caso de Hypoprion isodus, esta incerteza se debe a la existencia de una descripción original similar a $H$. heterodus, basada en algunas piezas dentales, que permanece sin aclararse y no ha permitido avanzar en su reconocimiento (Pequeño 1989). Pese a ello, es muy probable que por las descripciones anteriores a Philippi (Compagno 1984b), esta especie sea sinónimo de alguna especie del género Carcharhinus. Por otro lado, $H$. heterodus fue descrita sobre la base de un ejemplar del cual sólo se preservó una mandíbula con dientes (Fowler 1945), depositada en el Museo Nacional de Historia Natural en Santiago de Chile y de acuerdo con la descripción dentaria de esta especie, único carácter dibujado por Philippi, se supone que correspondería a Carcharhinus obscurus (Le Sueur, 1818) (Pequeño 1989). 
Geográficamente, se destaca el primer hallazgo de Echinorhinus cookei Pietschmann, 1928 (Echinorhinidae) en aguas salvadoreñas, registro que permite extender su rango de distribución conocida hasta hoy para el Pacífico suroriental (Rojas et al. 2006). Por otra parte, la captura de Sphyrna zygaena (Linnaeus, 1758) (Sphyrnidae) en la bahía de San Antonio (33³5' S) (Brito 2004b), aumentando su rango de distribución, ya que sólo hay antecedentes de su captura entre Arica (18 30' S) e Iquique (20 $12^{\prime} \mathrm{S}$ ) (Mann 1954, Sielfeld \& Vargas 1992).

Un aspecto destacable, es el aumento en el número de especies que ha experimentado la familia Dalatiidae en aguas chilenas debido a nuevos registros. Un caso es Etmopterus brachyurus Smith \& Radcliffe, 1912 cuya captura fue efectuada en las cercanías de las Islas Desventuradas en la cordillera sumergida de Nazca cerca de los 2556,6’S, 84²5,6’W (Oñate \& Pequeño 2005), ampliando su rango de distribución a gran parte de la cuenca del Océano Pacífico. También la captura de Etmopterus unicolor (Engelhardt, 1912) frente a las costas de Valdivia, constituye un nuevo integrante para la condrictiofauna chilena (Reyes \& Hüne 2006). Otro importante registro, es el de los ejemplares de Centroscyllium nigrum Garman, 1899 capturados en el Pacífico colombiano (Rubio et al. 2005) extendiendo así su rango de distribución geográfica. Relevante ha sido la captura de tres ejemplares de Heteroscymnoides marleyi Fowler, 1934 (Stehmann et al. 1999), uno de ellos capturado en el Pacífico sureste $\left(32^{\circ} 35,7^{\prime} \mathrm{S}, 85^{\circ} 25,2^{\prime} \mathrm{W}\right)$, registro que corresponde al denominado Mar Presencial de Chile. Otra especie ha de ser considerada es Scymnodalatias oligodon descrita por Kukuyev \& Konovalenko (1988), los cuales informan una captura del holotipo en 1985 en el Pacífico sureste (33 ${ }^{\circ}$ 31'S, 96 07'W). Finalmente, Somniosus pacificus Bigelow \& Schroeder, 1944 ha sido capurado frente a Valdivia (3945’S) (Pequeño et al. 1991) y frente a San Antonio, lo que permite aumentar su distribución geográfica (Brito 2004a).

A nivel de Familia, se adiciona la familia Pseudocarchariidae al orden Lamniformes, con una sola especie, Pseudocarcharias kamoharai (Matsubara, 1936), representada por un ejemplar capturado en la costa norte de Chile (Meléndez et al. 2006). Esto permite documentar la presencia de un nuevo taxón para aguas chilenas y geográficamente, indica la amplia distribución austral de esta especie en el Pacífico suroriental. Finalmente, la familia Alopiidae se enriquece con un nuevo registro en las costas chilenas con Alopias superciliosus (Lowe, 1841) capturada en aguas insulares y continentales ( $\left.24^{\circ} 07^{\prime} \mathrm{S}, 37^{\circ} 13^{\prime} \mathrm{S}\right)$, como fauna acompañante del pez espada Xiphias gladius Linnaeus (Acuña et al. 2002).

En la actualidad el Superorden Squalomorphi consta de 56 especies en Chile, agrupadas en seis órdenes y 16 familias (Anexo 1). En el presente trabajo se ha elaborado una clave taxonómica de reconocimiento de este grupo, incluyendo una breve descripción biogeográfica, indicando la distribución geográfica mundial y en Chile, con información de los registros de captura de las 56 especies chilenas consideradas, como base para el estudio y diagnosis del Superorden.

\section{Material Y MÉtodos}

La clave estuvo basada en los caracteres diagnósticos señalados en la literatura revisada, para el reconocimiento de familias, géneros y especies, considerando las siguientes referencias: Regan (1908a, b), Fowler (1951), Hubbs (1951), Smith (1957), Siccardi (1960), Garrick (1967), Arrizaga (1969-1970), Iwasaki (1970), Williams (1970), Gosztonyi (1973), Sadowsky (1973), Gubanov \& Grigor'ev (1975), Guzmán \& Campodónico (1976), Morillas (1977), Pequeño (1977), Nakaya \& Bass (1978), Flores \& Rojas (1979), Pequeño (1979a, b), Pequeño (1981), Siccardi et al. (1981), Wolfson \& Notarbartolo di Sciara (1981), Garrick (1982), Compagno (1984a, b), Dolganov (1984), Ruíz \& Fernández (1984), Meléndez \& Meneses (1986), Taniuchi \& Garrick (1986), Compagno (1988), Moreno et al. (1989), Pequeño (1989), Pequeño et al. (1990, 1991), Lloris \& Rucabado (1991), Sielfeld \& Vargas (1992), Meléndez et al. (1993), Meléndez \& Céspedes (1996), Long (1997), Pequeño (1997), Chirichigno \& Vélez (1998), Stehmann et al. (1999), Compagno (2001), Ríos et al. (2002), Cousseau \& Perrota (2003), Pequeño \& Sáez (2003), Brito (2004a, b), Pequeño \& Sáez (2004), Compagno et al. (2005), Menni \& Lucifora (2007), Parin \& Kotlyar (2007), Andrade \& Pequeño (2008), Eschmeyer \& Fricke (2010). Las sinonimias no se incluyen en la clave por estar indicadas en la literatura correspondiente.

Por razones prácticas y didácticas, la presentación se realizó sobre la base de varias claves, que permiten ir directamente al orden y reconocer un ejemplar. Se incluyó una clave para reconocer los órdenes y otra para reconocer familias, géneros y especies dentro de cada orden, para facilitar el reconocimiento de las características taxonómicas e identificación taxonómica de Squaliformes. Se incluyó un breve glosario de términos utilizados (Anexo 2). Para complementar los términos utilizados en la 
morfología externa de los tiburones, se recomienda consultar Chirichigno (1974), Compagno (1984a, b, 1988, 2001) y Chirichigno \& Vélez (1998).

\section{Resultados}

Según el análisis de la literatura científica mencionada y observaciones seleccionadas, se preparó la siguiente serie de claves taxonómicas, resumiendo los conocimientos sobre distribución geográfica de las especies (Tablas 1 y 2).

\section{Clave 1: Ordenes del Superorden Squalomorphi}

1 (2) Sin aleta anal. .3

2 (1) Con aleta anal. .5

3 (4) Cuerpo deprimido dorso centralmente, parecido a una raya, boca terminal. SQUATINIFORMES:

- Ojo pequeño, barbo nasal angosto, muesqueado y convexo, espacio internasal notablemente menor que el espacio interorbital (Pacífico este; Chile: Arica, Coquimbo, Puerto Montt a Estrecho de Magallanes)..... ..SQUATINIDAE. .Rhina armata

4 (3) Cuerpo no deprimido dorso ventralmente, no parecido a una raya, boca ventral

SQUALIFORMES. .Ver Clave 3

5 (6) Seis o siete pares de hendiduras branquiales, con una aleta dorsal........HEXANCHIFORMES...........Ver Clave 2

6 (5) Cinco pares de hendiduras branquiales, con dos aletas dorsales.

7 (8) Boca ventral (por debajo del hocico). .9

8 (7) Boca terminal (sin hocico definido); ojo por detrás del borde de la boca; dientes muy pequeños.

ORECTOLOBIFORMES:

- Hocico sobresale levemente de la boca; bordes longitudinales a lo largo del cuerpo; con líneas blancas longitudinales y verticales; presencia de manchas blancas en el cuerpo y aletas (Atlántico occidental y oriental, Indo Pacífico, Pacífico; Chile: Arica a Iquique y Constitución)... ...RHINCODONTIDAE ...Rhincodon typus

9 (10) Con membrana nictitante

CARCHARINIFORMES .Ver Clave 4

10 (9) Sin membrana nictitante ........LAMNIFORMES . ..Ver Clave 5

\section{Clave 2: Familias, géneros y especies del ORDEN HeXANCHIFORMES}

1 (2) Con seis pares de hendiduras branquiales; cuerpo elongado, anguiliforme; boca casi terminal; extremos inferiores de la primera hendidura branquial conectado al otro lado a través de la garganta. (Atlántico este, Océano Indico, Pacífico oeste, Pacífico este; Chile: San Vicente, Golfo de Arauco) ..CHLAMYDOSELACHIDAE.... .Chlamydoselachus anguineus

2 (1) Con seis a siete pares de hendiduras branquiales; cuerpo robusto, sin forma de anguila, boca ventral; primeras hendiduras branquiales no unidas a la garganta

HEXANCHIDAE .3

3 (4) Con seis hendiduras branquiales (Atlántico oriental, Indo-Pacífico, costa sudafricana, Pacífico occidental, central y oriental; Chile: Arica a Valdivia, Mehuín, isla Maiquillahue, Chiloé). Hexanchus griseus

4 (3) Con siete hendiduras branquiales. .5

5 (6) Ojos muy grandes, cabeza extremadamente angosta y puntiaguda. Cuerpo sin manchas, de tamaño pequeño (cerca de 1,4 m de longitud total) (Pacífico occidental y oriental; Chile: Arica, Cartagena, Coquimbo y Concepción) Heptranchias perlo

6 (5) Ojos pequeños, cabeza ancha y redondeada. Cuerpo generalmente con pequeñas manchas negras esparcidas y a veces también con manchas blancas, de tamaño grande (cerca de 2,9 m de longitud total) (Océano Indico occidental, Pacífico occidental y oriental; Chile: Chile central, Mehuín y Estrecho de Magallanes). .Notorynchus cepedianus

\section{Clave 3: Familias, gÉNERos y ESPECIES DEL ORdEN SQUALIFORMES}

1 (2) Hocico elongado a moderadamente elongado ..CENTROPHORIDAE..........3

2 (1) Hocico no elongado. .5

3 (4) Hocico espatulado, extremadamente largo, deprimido. Longitud del hocico preoral más grande que la distancia desde la boca al origen de las aletas pectorales. Primera aleta dorsal larga y baja, se origina por sobre las bases de las aletas pectorales; primera aleta dorsal con una espina más chica que aquella de la segunda aleta dorsal. Cuerpo café grisáceo (Atlántico noreste; Chile norte y central, Corral, Corcovado y Morro Yeli) Deania calcea

4 (3) Hocico no espatulado y moderadamente elongado, ampliamente parabólico. Longitud del hocico preoral menos o raramente igual a la distancia desde la boca al origen de 
las aletas pectorales. Primera aleta dorsal alta, se origina por sobre el margen interno de la aleta pectoral; espina de la segunda aleta dorsal más grande que aquella de la primera aleta dorsal (Atlántico este, oeste del Océano Indico, Pacífico este, Pacífico sur oriental; Chile: golfo de Ancud) .Centrophorus squamosus

5 (6) Primera aleta dorsal por detrás del origen de las aletas pélvicas (Pacífico oeste, Pacífico este; Chile: Arica, isla Salas y Gómez, San Antonio, Santo Domingo y golfo de Arauco)........ECHINORHINIDAE.....Echinorhinus cookei

6 (5) Primera aleta dorsal por delante del origen de las aletas pélvicas DALATIIDAE............7

7 (8) Espinas presentes al frente de ambas aletas dorsales . .9

8 (7) Sin espinas al frente de las aletas dorsales. .15

9 (10) Dientes sin cúspides o cúspides poco notorias en ambas mandíbulas (Pacífico este, Perú; Chile: Punta Carrranza, Taltal, Caldera, Valparaíso-San Antonio, Concón, Constitución y Corral) Aculeola nigra

10 (9) Dientes con cúspides en ambas mandíbulas...........11

11 (12) Dientes en la mandíbula superior con una cúspide .Centroscymnus.......29

12 (11) Dientes en la mandíbula superior con tres o más cúspides

13 (14) Dientes asimétricos en la mandíbula inferior, con una cúspide oblicua, dientes superiores con tres a cinco cúspides.

Etmopterus. .33

14 (13) Dientes similares en ambas mandíbulas; tres a cinco cúspides erectas y simétricas .Centroscyllium......47

15 (16) Extremo de la base de la primera aleta dorsal sobre la ventral (Atlántico, Pacífico, golfo de Guinea; Chile: Isla de Pascua) .Isistius brasiliensis

16 (15) Extremo de la base de la primera aleta dorsal al frente de la ventral.

17 (18) Primera aleta dorsal alejada de la segunda dorsal y cerca de la aleta pectoral

18 (17) Primera aleta dorsal muy cerca de la segunda dorsal y alejada de la aleta pectoral.

19 (20) Inserción de la primera aleta dorsal sobre la base de la aleta pectoral (Atlántico sur y central, Indico, Pacífico sureste). Heteroscymnoides marleyi

20 (19) Inserción de la primera aleta dorsal claramente por detrás de las bases de las aletas pectorales.
21 (22) Primera aleta dorsal sobre la mitad del dorso; segunda aleta dorsal más pequeña que la primera dorsal; aleta caudal semisimétrica y en forma de "raqueta", con un lóbulo superior relativamente corto y un lóbulo inferior grande (Pacífico norte; Cordillera sumergida de Nazca; Chile: San Antonio y Valdivia) Somniosus pacificus

22 (21) Primera aleta dorsal ligeramente posterior sobre el dorso; segunda aleta dorsal más grande que la primera dorsal; aleta caudal asimétrica sin forma de "raqueta", con un lóbulo superior grande y un lóbulo inferior corto (Pacífico sur oriental)..................Scymnodalatias oligodon

23 (24) Base de la segunda aleta dorsal mayor que la longitud de la base de la primera dorsal......................25

24 (23) Base de la segunda aleta dorsal igual que la longitud de la primera dorsal (Cordillera sumergida de Nazca) .Mollisquama parini

25 (26) Aleta pectoral redondeada, espinas predorsales pequeñas.

26 (25) Aleta pectoral no redondeada, espinas predorsales grandes. .SQUALIDAE. .51

27 (28) Cabeza y cuerpo negro, aletas con prominentes áreas blancas y transparentes in vivo (Atlántico sur central, Indico, Pacífico sur central, Pacífico norte central, Pacífico sur; sur de Chile)...

.Euprotomicrus bispinatus

28 (27) Cuerpo uniformemente negro, aletas sin prominentes áreas blancas (Pacífico oeste: Japón, costa sureste de Australia, Nueva Zelanda; Chile: Iquique, Antofagasta, Copiapó, Los Vilos, Concepción, golfo de Arauco, golfo de Ancud, isla Linguar y Punta Gruesa) .Zameus squamulosus

29 (30) Pliegues labiales superiores muy largos (Atlántico noreste, Indico; Chile: norte de Chile, isla Mocha y Corral) Centroscymnus crepidater

30 (29) Pliegues labiales superiores muy cortos .31

31 (32) Segunda aleta dorsal considerablemente más grande que la primera; dentículos del tronco relativamente grandes, con la mayor parte de los bordes suaves y ovales. Cuerpo café oscuro (Atlántico norte occidental, Pacífico occidental: Japón, Nueva Zelanda, Australia; Chile: isla Salas y Gómez, cordillera sumergida de Nazca)....Centroscymnus owstonii

32 (31) Segunda aleta dorsal algo más alta que la primera; dentículos laterales del tronco grandes y en los bordes con la presencia de tres cúspides. Cuerpo café oscuro o negruzco (Atlántico sur occidental; Chile: Corral, entrada oriental Estrecho de Magallanes).....Centroscymnus macracanthus 
33 (34) Dentículos sin espinas (Atlántico, Japón, Nueva Zelanda, Natal; Chile: cordillera sumergida de Nazca, isla Salas y Gómez, sur de Chile)..............Etmopterus pusillus

34 (33) Dentículos con espinas. . .35

35 (36) Dentículos cónicos dispuesto en hileras sobre el cuerpo .37

36 (35) Dentículos cónicos no dispuestos en hileras sobre el cuerpo. .39

37 (38) Primera aleta dorsal por detrás de los bordes libres de las aletas pectorales; espacio interdorsal corto; dientes superiores con menos de tres pares de cúspides laterales (Pacífico norte, Pacífico suroriental; Chile: Valdivia) Etmopterus unicolor

38 (37) Primera aleta dorsal por delante de los bordes libres de las aletas pectorales; espacio interdorsal grande; dientes superiores con una a tres cúspides laterales (Perú; Chile: cordillera sumergida de Nazca, isla Salas y Gómez) ..Etmopterus pycnolepis

39 (40) Aberturas branquiales largas. . .43

40 (39) Aberturas branquiales cortas. . .41

41 (42) Cuerpo moderadamente grueso, primera aleta dorsal levemente al frente de las aletas pectorales, segunda aleta dorsal más grande que la primera dorsal; dientes superiores con menos de tres pares de cúspides laterales (Atlántico sur occidental y oriental, Pacífico suroriental; Chile: cordillera sumergida de Juan Fernández, Corral, Aysén al sur).

Etmopterus granulosus

42 (41) Cuerpo muy delgado, primera aleta dorsal encima de las aletas pectorales, segunda aleta dorsal mucho más grande que la primera dorsal; dientes superiores con tres pares de cúspides laterales (Natal, sur de Mozambique, Filipinas, Bali y Australia; Chile: cordillera sumergida de Nazca). Etmopterus brachyurus

43 (44) Dientes de la mandíbula superior con una a tres cúspides laterales (Pacífico suroriental: Perú; Chile: cordillera sumergida de Nazca, isla Salas y Gómez) ..Etmopterus litvinovi

44 (43) Dientes de la mandíbula superior con menos de tres cúspides laterales. .45

45 (46) Primera aleta dorsal situada levemente por detrás de las aletas pectorales, segunda aleta dorsal más grande que la dorsal (Atlántico suroccidental, Indico occidental, Pacífico occidental; Chile). Etmopterus lucifer

46 (45) Primera aleta dorsal situada por sobre las aletas pectorales, segunda aleta dorsal mucho más grande que la primera dorsal (Pacífico central, islas Hawaianas; Chile: Caldera). .Etmopterus villosus

47 (48) Extremo posterior de las terminaciones de las aletas pectorales muy anterior al origen de la primera espina dorsal. Distancia entre las bases de las aletas pectorales y pélvicas mucho más grande que la longitud de la cabeza. .49

48 (47) Extremo posterior de las terminaciones de las aletas pectorales casi opuesta al origen de la primera espina dorsal, levemente al frente de éste. Distancia entre las bases de la aleta pectoral y pélvica casi igual o menos que la longitud de la cabeza. Squalus......51

49 (50) Segunda aleta dorsal más grande que la primera; cuerpo moderadamente robusto y comprimido. Cuerpo de color café oscuro por encima y por debajo; aletas claras (Atlántico suroeste, Islas Malvinas; Chile: Arica a Estrecho de Magallanes y Canal Beagle).

..Centroscyllium granulatum

50 (49) Segunda aleta dorsal casi tan larga como la primera; cuerpo robusto y comprimido. Cuerpo café grisáceo arriba, abruptamente negruzco a los lados inferiores de la cabeza y abdomen, con una banda negra en el lado inferior del pedúnculo caudal extendiéndose sobre las bases de las aletas pélvicas (estas áreas negras indicarían presencia de fotóforos); aletas de bordes blancos (Pacífico norte; Chile: costa del norte y centro, cordillera sumergida de Juan Fernández, Punta Nogurue a Chiloé)

Centroscyllium nigrum

51 (52) Cuerpo delgado. Hocico subangular, aguzado, estrecho y moderadamente largo. Primera aleta dorsal situada posteriormente, originándose por sobre o por detrás de los extremos libres de la pectoral; origen de la espina por detrás de los extremos (Atlántico oeste, Atlántico occidental y oriental, Pacífico occidental y oriental; Chile: Arica, isla San Ambrosio, archipiélago y cordillera sumergida de Juan Fernández, Corral y Cabo de Hornos) .Squalus acanthias

52 (51) Cuerpo robusto. Hocico redondeado parabólico, ancho y moderadamente largo. Primera aleta dorsal situada más anteriormente, con origen casi por sobre las inserciones de la pectoral; origen de la espina por sobre los márgenes internos de la pectoral y bien al frente de sus extremos libres. .53

53 (54) Primera espina predorsal larga, casi o un poco más larga que la base de la aleta; segunda espina predorsal muy larga, levemente más alta que la aleta. (Atlántico oriental, Pacífico occidental, Indico). Squalus blainville 
54 (53) Primera espina predorsal moderadamente larga, mucho menos que la longitud de la base de la aleta; segunda espina predorsal larga, casi tan alta como la aleta. (Cosmopolita, archipiélago y cordillera sumergida de Juan Fernández) Squalus mitsukurii

\section{Clave 4: Familias, gÉneros y ESPECIES del ORden CARCHARINIFORMES}

1 (2) Cabeza aplanada y expandida lateralmente formando una estructura en forma de martillo.....SPHYRNIDAE....29

2 (1) Cabeza no aplanada ni expandida. . .3

3 (4) Primera aleta dorsal sobre o por detrás del origen de las aletas pélvicas ..SCYLIORHINIDAE....7

4 (3) Primera aleta dorsal por delante del origen de las aletas pélvicas . .5

5 (6) Ausencia de fosa precaudal; presencia de espiráculo; segunda aleta dorsal casi de igual tamaño que la primera aleta dorsal (excepto en Galeorhinus.....TRIAKIDAE...19

6 (5) Presencia de fosa precaudal; ausencia de espiráculo o muy diminuto; segunda aleta dorsal mucho más pequeña que la primera aleta dorsal.........CARCHARHINIDAE..25

7 (8) Cabeza larga, ancha y aplanada; origen de la primera aleta dorsal anterior o ligeramente anterior al origen de las aletas pélvicas; hendiduras branquiales muy espaciadas (Pacífico oriental: sur de Baja California y Golfo de California, México; Chile: norte de Chile y sur de Coquimbo)

..Cephalurus cephalus

8 (7) Cabeza más corta; origen de la primera aleta dorsal posterior al origen de las aletas pélvicas; hendiduras branquiales juntas.

9 (10) Ausencia de surcos labiales en las esquinas de la boca; presencia de cresta o borde supraorbital (Pacífico oriental: California central a Golfo de California y sur de México; Pacífico sur: Chile central)

Cephaloscyllium ventriosum

10 (9) Presencia de surcos labiales continuos alrededor de las esquinas de la boca; ausencia de cresta supraorbital (excepto Schroedericthys chilensis) .11

11 (12) Hocico largo, poros muy notorios en la superficie ventral; cabeza aplanada y espatulada. . .13

12 (11) Hocico corto, poros poco notorios en la superficie ventral. .15

13 (14) Espacio interdorsal casi igual al hocico preoral (Pacífico oriental: British Columbia, Canadá, Baja
California, México, sur de Panamá, Ecuador, Perú; Chile: Valparaíso-San Antonio y bahía Chanco)

Apristurus brunneus

14 (13) Espacio interdorsal mayor que el hocico preoral (Pacífico sur; Chile: Antofagasta, Valparaíso, golfo de Arauco, Corral y Punta Angeles)...........Apristurus nasutus

15 (16) Dorso sin manchas (Pacífico suroriental, Perú; Chile: Arica, Algarrobo, Los Vilos, Pichidangui, Corral y Cabo de Hornos). Bythaelurus canescens

16 (15) Dorso con manchas .17

17 (18) Numerosas manchas o marcas obscuras, con trazos de bandas verticales; los surcos labiales de las mandíbulas superior e inferior muy largos, sus longitudes similares al diámetro ocular (Atlántico sur y Pacífico oriental; Argentina, Perú; Chile: Arica, La Herradura, El Tabo, San Antonio, Matanzas, Constitución, Mehuín, Curiñanco, Corral, Chiloé, Morro Yeli y Puerto Natales) Schroederichthys chilensis

18 (17) Manchas oscuras (siete), en forma de triángulo invertido sobre el dorso; un surco labial alrededor de la comisura bucal, extendiéndose a cierta distancia. Manchitas claras, entre y sobre las aletas pectorales y pélvicas. Región ventral y parte inferior de la cabeza, blancuzca (Atlántico sur occidental, sur de Argentina a Estrecho de Magallanes, Pacífico sur; Chile: Valparaíso, Corral, frente a Mehuín y Curiñanco, bahía Carnero, Morro Yeli, Punta Arenas y Cabo de Hornos). Schroederichthys bivius

19 (20) Segunda aleta dorsal notoriamente más pequeña que la primera; lóbulo inferior de la aleta caudal casi la mitad de la longitud del margen dorsal de la caudal (Angola, este de Londres, Natal, Atlántico norte y Mediterráneo, Pacífico; Chile: Arica, Valdivia, Playa Chan Chan y Morro Bonifacio). Galeorhinus galeus

20 (19) Segunda aleta dorsal casi tan larga como la primera; lóbulo inferior de la caudal ausente a corto en ejemplares adultos, débilmente marcado o ausente en juveniles.......21

21 (22) Dientes monocuspidiales o molariformes.

Mustelus.

22 (21) Dientes con dos a tres cúspides bajas, triangulares y asimétricas (California hasta Chile: Arica, Playa Brava, Iquique)..... Triakis maculata

23 (24) Cabeza larga, cuerpo levemente robusto, dientes cuspidiados y asimétricos, con una prominente cúspide primaria y ocasionalmente con cúspides secundarias bajas; cuerpo gris o café grisáceo abajo, claro por arriba, sin 
manchas en el cuerpo. Desarrollo vivíparo (Pacífico suroriental; Perú, Chile: sur de Chile)....Mustelus whitneyi

24 (23) Cabeza corta, cuerpo robusto, dientes sin cúspides y simétricos; cuerpo gris entero o café grisáceo arriba, claro por abajo, con numerososas manchas blancas; presencia de barras obscuras en juveniles de $35 \mathrm{~cm}$. Desarrollo ovovíviparo (Atlántico suroriental, Pacífico suroriental: Islas Galápagos, Perú; Chile: Arica, Archipiélago de Juan Fernández, Corral y extremo sur de Chile).

...Mustelus mento

25 (26) Origen de la primera aleta dorsal sobre o levemente por delante de los extremos libres de las aletas pectorales pero siempre más cerca de éstos que del punto de inserción........................................Carcharhinus...27

26 (25) Origen de la primera aleta dorsal muy atrás; el punto medio de su base mucho más cerca al origen de las aletas pectorales que a la axila de la aleta pectoral; cuerpo de color azul intenso. (Areas tropicales, sureste costa Ciudad del Cabo; Chile: Archipiélago de Juan Fernández, Valdivia y Pisagua). Prionace glauca

27 (28) Primera dorsal de tamaño moderado y semifalcada, origen primera aleta dorsal usualmente sobre o levemente anterior a los extremos libres de las pectorales. Pectorales grandes y falcadas. Hocico corto a moderadamente largo y ampliamente redondeado. Dientes superiores anchos, triangulares y fuertemente serrados; aleta pectorales no rectas; borde anterior de la primera aleta dorsal levemente curvado (Atlántico occidental, Atlántico noreste, Indico occidental, Pacífico occidental, Pacífico oriental; Chile)... .Carcharhinus obscurus

28 (27) Primera dorsal moderadamente grande y falcada, origen primera dorsal sobre la longitud media de los márgenes internos de la pectoral. Pectorales grandes y semifalcada. Hocico moderadamente largo y ampliamente redondeado. Dientes ánterolaterales superiores relativamente altos y angostos; aletas pectorales casi rectas; borde anterior de la primera dorsal casi recto (Circumtropical, Atlántico norte occidental, central y oriental, Indico suroriental, Pacífico central occidental, Pacífico oriental, Islas Oceánicas; Chile: Isla de Pascua, isla Salas y Gómez).................Carcharhinus galapagensis

29 (30) Margen anterior de la cabeza dividido en tres lóbulos, la línea media de la cabeza redondeada (en ejemplares grandes es más recta), no indentada en el centro; base de la aleta anal casi igual o un poco más larga que la base de la segunda aleta dorsal (Atlántico oriental y norte, Indico occidental, Pacífico central y oriental; Chile: Arica a Iquique y San Antonio)... Sphyrna zygaena
30 (29) Margen anterior de la cabeza dividido en cuatro lóbulos, la línea media de la cabeza indentada; base de la aleta anal más larga que la base de la segunda dorsal (Circumglobal. Atlántico Norte; Pacífico, Indico, central y oriental, Pacífico sur; Chile: Caldera y Paico)

Sphyrna lewini

\section{Clave 5: FAMILIAS, GÉNEROS Y ESPECIES DEL ORDEN LAMNIFORMES}

1 (2) Longitud de la aleta caudal casi igual que la longitud del cuerpo. ..ALOPIIDAE..........3

2 (1) Longitud de la aleta caudal no es igual que la longitud del cuerpo. .5

3 (4) Cabeza con una cresta prominente que se extiende desde el nivel de los ojos hacia abajo y atrás, soprepasando las hendiduras branquiales; menos de 15 dientes en ambas mandíbulas. Ojos grandes, extendiéndose sobre la superficie de la cabeza; primera aleta dorsal muy atrás, su extremo posterior sobre o a nivel del origen de las aletas pélvicas (Atlántico occidental y oriental, Indico, Pacífico, Chile insular). .Alopias superciliosus

4 (3) Cabeza sin cresta prominente o una ligera indentación que corre desde el nivel de los ojos hasta las hendiduras branquiales; más de 15 dientes en ambas mandíbulas; el extremo posterior de la primera aleta dorsal muy delante de las pélvicas (Atlántico occidental y oriental, Indico, Pacífico central y suroriental; Chile: Arica, Antofagasta, San Antonio, Talcahuano y Valdivia)......Alopias vulpinus

5 (6) Pedúnculo caudal con quillas laterales fuertes..........7

6 (5) Pedúnculo caudal sin quillas laterales o débiles (Atlántico occidental, Indico oriental, Pacífico oriental; Chile: Valdivia). PSEUDOCARCHARIIDAE. Pseudocarcharias kamoharai

7 (8) Hendiduras branquiales muy largas, extendiéndose desde arriba del dorso hasta cerca de la línea media del lado ventral o mitad de la garganta (Atlántico occidental, Indico oriental, Pacífico oriental; Chile: Arica, Talcahuano y Corral).....................................ETORHINIDAE....... Cethorhinus maximus

8 (7) Hendiduras branquiales no se extienden hasta la superficie inferior del cuerpo, sólo están restringidas a los lados del cuerpo; sin branquispinas; dientes grandes en poco número. ..LAMNIDAE . .9

9 (10) Dientes aserrados, los superiores son planos y con cúspides anchas y triangulares, fuertes quillas en el pedúnculo caudal (Cosmopolita, Atlántico occidental y 
oriental, Pacífico occidental central y oriental; Chile: Arica, Iquique, Valparaíso y Talcahuano).

Carcharodon carcharias

10 (9) Dientes no aserrados, los superiores no son aplanados y con cúspides triangulares.

11 (12) Origen de la primera dorsal sobre o detrás de la inserción de la aleta pectoral; origen de la aleta anal por debajo del origen la segunda dorsal (Cosmopolita, Atlántico occidental y oriental, Pacífico suroriental, Subantártico; Chile: Arica, San Antonio, Valdivia y extremo sur del país).

Lamna nasus

12 (11) Origen de la primera aleta dorsal por detrás de los extremos libres de las aletas pectorales. Origen de la aleta anal bajo o levemente posterior a la segunda dorsal. Sin quillas secundarias en la aleta caudal. Isurus....13

13 (14) Hocico usualmente puntiagudo. Aleta pectoral considerablemente más cortas que la cabeza. Lado ventral del hocico y boca de color blanco (Atlántico occidental y oriental, Atlántico este, Indico, Pacífico central y oriental; Chile: Arica, San Antonio y Valdivia)....Isurus oxyrinchus

14 (13) Hocico usualmente no aguzado. Aletas pectorales tan grandes como la cabeza. Lado ventral del hocico y boca oscuro (Atlántico norte, Pacífico central, Pacífico suroriental; Chile: Caldera y Paico). .Isurus paucus

Es importante destacar que en algunos géneros hay dos o más especies y que solamente una se encuentra en aguas chilenas. En otros casos se produce la situación que dentro de un género hay agrupaciones de especies (no necesariamente descritas como subgénero) y en Chile se encuentra sólo una de las especies. Además en la literatura utilizada (ver citas en Material y métodos) entregan características taxonómicas complementarias de nivel específico, cuando hay ejemplares que presentaron dudas clasificatorias o no son suficientes los datos aportados por la clave precedente, por ejemplo:

- Chlamydoselachus anguineus: Cuerpo delgado y con prominentes quillas en el abdomen; hocico extremadamente corto, truncado, boca muy larga; dientes tricuspidiados en ambas mandíbulas. Aleta anal grande, más grande que la dorsal, aleta caudal sin muesca subterminal.

- Hexanchus griseus: Boca chica, ancha. Base de la aleta dorsal separada desde el margen superior del origen de la aleta por una distancia aproximadamente igual o ligeramente más grande que su longitud.
- Echinorhinus cookei: Pequeños dentículos del cuerpo uniformemente distribuidos, no están fusionados en placas o escudos y en grupos de tres a cinco.

- Aculeola nigra: Espinas de las aletas muy cortas. Cabeza con alerones nasales muy cortos; alerones no expandidos como barbillas, hocico corto, aplanado y truncado.

- Isistius brasiliensis: Extremos de los lóbulos de la aleta caudal negruzcos. Dorso, por lo común café oscuro; ventralmente claramente pálido, excepto por unas bandas negruzcas presentes a través del pecho.

- Heteroscymnoides marleyi: Primera aleta dorsal más larga que la segunda, ojos grandes (cavidad ampliamente oval a semicircular con un borde superior recto); hendiduras branquiales cortas, la quinta justo al frente del origen de la aleta pectoral; cuerpo café.

- Somniosus pacificus: Cuerpo cilíndrico. Cabeza redonda y chica, hocico corto, redondeado. Perfil dorsal no convexo. Dientes superiores lanceolados, dientes inferiores con cúspides oblicuas. Diámetro vertical de la órbita más chico que la altura mínima del pedúnculo caudal.

- Scymnodalatias oligodon: Cuerpo elongado. Cabeza grande cónica. Hocico grande, cónico, dorsoventralmente aplanado. Perfil dorsal del cuerpo más convexo que la ventral. Dientes superiores inclinados hacia los bordes de la boca, dientes inferiores con cúspides como aguja. Diámetro vertical de la órbita más chico que la profundidad del pedúnculo caudal.

- Mollisquama parini: Cuerpo elongado, levemente comprimido a los lados, cubierto de escamas excepto en una estrecha banda en el borde posterior de las aletas; escamas pequeñas, delgadas en forma de hoja; aletas pectorales pequeñas (con una glándula en mitad posterior de ambas aletas, con función desconocida); dientes superiores monocuspidiales, dientes de la mandíbula inferior tricuspidiados. Cuerpo café oscuro con pequeñas manchas tenues en el dorso y costados.

- Centroscymnus crepidater: Ambas dorsales casi de la misma altura; dentículos del tronco moderadamente grandes, anteriormente con bordes suaves, pero posteriormente ovales y con coronas cuspidiadas. Cuerpo negro o café.

- Etmopterus pusillus: Dentículos con coronas bajas, aplanadas, cóncavas, sésiles, sin ninguna espina. Piel suave comparada con otras especies del género. Cuerpo café grisáceo obscuro dorsal y ventralmente. 
- Etmopterus litvinovi: Segunda aleta dorsal ligeramente más grande que la primera dorsal; primera dorsal situada por detrás de las aletas pectorales.

- Squalus acanthias: Punto medio de la base de las pélvicas mucho más cerca del origen de la segunda dorsal que la inserción de la primera dorsal. Cuerpo color gris arriba, blanco por debajo, usualmente con notorias manchas blancas a ambos lados, dorsal y aleta sin bordes blancos.

- Bythaelurus canescens: Cuerpo negro grisáceo a pálido; el vientre más claro, sin manchas bien definidas; surcos labiales de ambas mandíbulas cortos, sus longitudes mucho menores que el diámetro ocular.

- Triakis maculata: Cuerpo con manchas pequeñas negras o gris a marrón uniforme con el extremo de las aletas blanco, redondeadas, más pequeñas y menos numerosas en ejemplares grandes; bordes de las aletas dorsales oscuros; primera aleta dorsal ancha, triangular y su margen posterior sesgado hacia atrás desde el ápex; aleta caudal sin lóbulo inferior bien marcado.

- Pseudocarcharias kamoharai: Cuerpo cilíndrico y delgado, cabeza corta más larga que el tronco, ojos grandes, sin branquispinas desarrolladas, dientes grandes en ambas mandíbulas.

- Cethorhinus maximus: Cuerpo fusiforme y moderadamente robusto, ojos chicos, con branquispinas muy desarrolladas; dientes pequeños y numerosos.

- Carcharodon carcharias: Cuerpo robusto sin quillas secundarias, superficie ventral blanca.

- Lamna nasus: Hocico relativamente grande. Presencia de cortas quillas secundarias en la base de la aleta caudal. Lado ventral blanco, sin manchas blancas, extremo libre de la primera aleta dorsal, notoriamente blanco.

\section{RASGOS BIOLÓGICOS Y DE DISTRIBUCIÓN GEOGRÁFICA DE LOS Tiburones de ChILE}

Tal como ha señalado Pequeño et al. (1990), existe una marcada diferencia entre los tipos de distribución geográfica de los Batidoidimorpha (rayas, en general) y los Squalomorpha (tiburones y similares), siendo los primeros generalmente bentónicos, mientras los segundos se consideran típicos pelágicos, con algunos grupos que son pelágico-demersales y escasos demersales-bentónicos.

Las características pelágicas de los tiburones, les exigen una alta capacidad y habilidad de natación y recorrer grandes distancias derivando una amplia distribución geográfica en algunos grupos. Es probable que en las amplias distribuciones, influya también la relativa mayor antigüedad de ciertas familias, como por ej. Chlamydoselachidae y Hexanchidae, que aunque poseen especies en número inferior a otras familias, sí están presentes en la mayoría de los océanos, como ocurre con Chlamydoselachus anguineus, Heptrachias perlo, Hexanchus griseus y Notorhynchus cepedianus. Entre las especies de tiburones típicamente pelágicos, se encuentran los tiburones más grandes conocidos, como aquellos de los géneros Rhincodon, Cetorhinus, Alopias, Charcharodon y Lamna, entre otros. Los individuos de estos grupos paren crías vivas y se reproducen en las diferentes regiones de su distribución geográfica, lo que contribuye al menos, a mantener esos rasgos de distribución.

Los tiburones netamente pelágicos tienen características morfológicas muy hidrodinámicas, lo cual no significa que otros (e.g., los demersales-bentónicos) no las tengan también. Sin embargo, las formas de las aletas caudales y los colores del cuerpo, entre otras características, delatan diferentes comportamientos natatorios.

Otras familias, con mayor número de especies, como Dalatiidae, han preferido determinados ambientes, tal como el engybentos (= demersal), existiendo algún grado de dependencia con respecto al fondo, principalmente por necesidades tróficas y reproductivas.

Familias como Scyliorhinidae, son más relacionadas con el fondo y sus especies son generalmente bentónicas y demersales, habitando profundidades que van desde la orilla misma (e.g., Schroederichthys y Halaelurus), hasta el talud continental y montes submarinos oceánicos (e.g., Etmopterus).

Estas familias, más dependientes del fondo, muestran formas menos adaptadas a la natación pelágica, tendientes más bien a una reptación acuática. Por otro lado, diversas especies de hábitos demersales a bentónicos, se reproducen mediante fecundación interna y huevos de gran tamaño comparados con otros peces y muchos otros vertebrados. Dichos huevos caen al fondo y la mayoría de las veces se enredan con macroalgas y es natural que las crías permanezcan en la misma zona donde viven sus padres y así, se genera un área de distribución geográfica relativamente limitada para tales especies. El ciclo de vida se desarrolla en un área bien delimitada, donde no sólo ocurre el proceso reproductivo en la forma descrita, sino también los tiburones pequeños - ya sin saco vitelino - se adaptan a comer ciertas presas, propias de ese rango de distribución geográfica, que los caracteriza. Este marco de ideas distributivo-funcionales, explica aunque sea 
Tabla 1. Especies de tiburones de distribución restringida al océano Pacífico sur oriental, con extensión hasta el sur de Argentina y otras regiones ( $\mathrm{X}=$ Presencia; - = Ausencia, ? = Presencia dudosa) / Shark species whose distribution restricted to the south eastern Pacific Ocean, stretch up to southern Argentina and other regions ( $\mathrm{X}=$ Presence, - =Absence, ? = Presence with doubt)

\begin{tabular}{|c|c|c|c|c|c|}
\hline Especie & Ecuador & Perú & Chile & Argentina & Otros datos biogeográficos \\
\hline 1) Aculeola nigra & $\mathrm{X}$ & $\mathrm{X}$ & $\mathrm{X}$ & - & \\
\hline 2) Bythaelurus canescens & $\mathrm{x}$ & $\mathrm{x}$ & $\mathrm{x}$ & - & \\
\hline 3) Centroscyllium granulatum & - & $\mathrm{x}$ & $\mathrm{x}$ & $\mathrm{x}$ & \\
\hline 4) Centroscymmus macracanthus & - & - & $\mathrm{X}$ & $\mathrm{X}$ & \\
\hline 5) Centroscymnus owstoni & - & - & $\mathrm{X}$ & - & Australia-Nueva Zelanda \\
\hline 6) Cephaloscyllium ventriosum & - & - & $\mathrm{x}$ & - & Bipolar (California) \\
\hline 7) Cephalurus cephalus & - & - & $\mathrm{X}$ & - & Bipolar \\
\hline 8) Etmopterus villosus & - & - & $\mathrm{x}$ & - & Hawaii \\
\hline 9) Isistius brasiliensis & $\mathrm{x}$ & $\mathrm{x}$ & $\mathrm{x}$ & - & Circumtropical \\
\hline 10) Mollisquama parini & - & - & $\mathrm{X}$ & - & \\
\hline 11) Mustelus mento & - & $\mathrm{x}$ & $\mathrm{x}$ & $?$ & \\
\hline 12) Mustelus whitneyi & $\mathrm{x}$ & $\mathrm{x}$ & $\mathrm{X}$ & - & \\
\hline 13) Zameus squamulosus & - & - & $\mathrm{X}$ & - & Australia \\
\hline 14) Schroederichthys bivius & - & $\mathrm{x}$ & $\mathrm{x}$ & $\mathrm{X}$ & Desde $25^{\circ} \mathrm{S}$ al sur \\
\hline 15) Schroederichthys chilensis & $\mathrm{x}$ & $\mathrm{x}$ & $\mathrm{x}$ & - & \\
\hline 16) Rhina armata & $\mathrm{X}$ & $\mathrm{X}$ & $\mathrm{X}$ & - & Canadá a México-California \\
\hline 17) Triakis maculata & $\mathrm{X}$ & $\mathrm{X}$ & $\mathrm{X}$ & - & \\
\hline
\end{tabular}

parcialmente, la distribución geográfica de los tiburones chilenos.

Las especies de tiburones que habitan entre Chile y California-Oregon tienen mayores similitudes biogeográficas que aquellos comunes entre Chile y Namibia (Sudáfrica). Aparentemente, en la cuenca del Pacífico, relativamente más antigua, se han configurado conjuntos que presentan similitudes más estrechas, que los vínculos geográficos que pudiesen tener con otras regiones, como es el caso de Namibia (Pequeño et al. 1990). Entre las especies de distribución restringida hay un mayor interés en las que se consideran endémicas. En el Pacífico sur oriental, que incluye el Mar de Chile, existen una serie de especies de tiburones que son endémicas, ciertas especies son comunes entre Chile y Ecuador y un número algo mayor con Perú y Argentina. Sólo una especie, Mollisquama parini
Dolganov, 1984, capturada únicamente en montes submarinos hacia el centro del océano Pacífico, sería endémica en mares de Chile (Tabla 1).

\section{Discusión}

En la vasta literatura de taxonomía y sistemática existente es frecuente encontrar algunos autores que tratan a las especies de tiburones sin detallar la distribución geográfica en Chile. (Chirichigno 1974, Compagno 1984a, 1984b, Chirichigno \& Vélez 1998, Compagno 2001).

Pese a que algunos autores chilenos han elaborado claves con descripciones y han citado los hallazgos de los ejemplares (Fowler 1951, Mann 1954, De Buen 1959, Meléndez \& Meneses 1986, Sielfeld \& Vargas 1992, Lamilla \& Bustamante 2005), en la actualidad, son escasas las referencias en Chile que integren los cambios 
Tabla 2. Especies de tiburones de distribución restringida al océano Pacífico sur oriental, en las cuales se requiere precisar su distribución en mares de Chile / Shark species of geographical distribution restricted to the southern eastern Pacific Ocean, whose precise distribution in Chilean seas needs to be confirmed

\begin{tabular}{|c|c|c|}
\hline Especie & Distribución en Chile & Referencia bibliográfica \\
\hline 1) Aculeola nigra & $\begin{array}{l}\text { Punta Carranza, Caldera, } \\
\text { Valparaíso-San Antonio, } \\
\text { Concón, Constitución, Corral }\end{array}$ & $\begin{array}{l}\text { Meléndez et al. (1993), } \\
\text { Andrade \& Pequeño (2008) }\end{array}$ \\
\hline 2) Bythaelurus canescens & $\begin{array}{l}\text { Arica, Algarrobo, Los Vilos, } \\
\text { Pichidangui, Cabo de Hornos }\end{array}$ & $\begin{array}{l}\text { Meléndez et al. (1993), } \\
\text { Compagno (1999) }\end{array}$ \\
\hline 3) Centroscyllium gramulatum & $\begin{array}{l}\text { Arica a Estrecho de Magallanes } \\
\text { y canal Beagle }\end{array}$ & $\begin{array}{l}\text { Meléndez et al. (1993), } \\
\text { Andrade \& Pequeño (2008) }\end{array}$ \\
\hline 4) Centroscymmus macracanthus & $\begin{array}{l}\text { Corral, entrada oriental del Estrecho } \\
\text { de Magallanes }\end{array}$ & $\begin{array}{l}\text { Compagno (1984a), } \\
\text { Compagno (1999) }\end{array}$ \\
\hline 5) Centroscymmus owstonii & $\begin{array}{l}\text { Isla Salas y Gómez, } \\
\text { cordillera sumergida de Nazca }\end{array}$ & $\begin{array}{l}\text { Pequeño (1989), } \\
\text { Compagno (1999) }\end{array}$ \\
\hline 6) Cephaloscyllium ventriosum & Chile central & $\begin{array}{l}\text { Compagno (1984b), } \\
\text { Schaaf-Da Silva \& Ebert (2008) }\end{array}$ \\
\hline 7) Cephalurus cephalus & Norte de Chile, sur de Coquimbo & $\begin{array}{l}\text { Meléndez et al. (1993), } \\
\text { Chirichigno \& Vélez (1998) }\end{array}$ \\
\hline 8) Etmopterus villosus & Caldera & $\begin{array}{l}\text { Pequeño (1989), } \\
\text { Compagno (1999) }\end{array}$ \\
\hline 9) Isistius brasiliensis & Isla de Pascua & $\begin{array}{l}\text { Compagno (1984a), } \\
\text { Compagno et al. }(2005)\end{array}$ \\
\hline 10) Mollisquama parini & Cordillera sumergida de Nazca & $\begin{array}{l}\text { Dolganov (1984), } \\
\text { Compagno (1999) }\end{array}$ \\
\hline 11) Mustelus mento & $\begin{array}{l}\text { Arica, archipiélago Juan Fernández, } \\
\text { Corral, extremo sur de Chile }\end{array}$ & $\begin{array}{l}\text { Mann (1954), } \\
\text { Compagno et al. (2005) }\end{array}$ \\
\hline 12) Mustelus whitneyi & Sur de Chile & $\begin{array}{l}\text { Compagno (1984a), } \\
\text { Pequeño (1997), } \\
\text { Chirichigno \& Vélez (1998), } \\
\text { Compagno (1999) }\end{array}$ \\
\hline 13) Scymnodon squamulosus & $\begin{array}{l}\text { Iquique, Antofagasta, Copiapó, } \\
\text { Los Vilos, Concepción, } \\
\text { golfo de Arauco, golfo de Ancud, } \\
\text { isla Linguar, Punta Gruesa }\end{array}$ & $\begin{array}{l}\text { Compagno (1984a), } \\
\text { Meléndez (1991), } \\
\text { Meléndez et al. (1993), }\end{array}$ \\
\hline 14) Schroederichthys bivius & $\begin{array}{l}\text { Valparaiso, Corral, Mehuín, } \\
\text { Curiñanco, bahia Carnero, } \\
\text { Morro Heli, Punta Arenas, } \\
\text { Cabo de Hornos }\end{array}$ & $\begin{array}{l}\text { Compagno (1984b), } \\
\text { Meléndez et al. (1993), } \\
\text { Chirichigno \& Vélez (1998), } \\
\text { Compagno (1999) }\end{array}$ \\
\hline 15) Schroederichthys chilensis & $\begin{array}{l}\text { Arica, La Herradura, El Tabo, } \\
\text { San Antonio, Matanzas, Constitución, } \\
\text { Mehuín, Curiñanco, Corral, Chiloé, } \\
\text { Morro Heli, Puerto Natales }\end{array}$ & $\begin{array}{l}\text { Meléndez et al. (1993), } \\
\text { Compagno et al. (2005) }\end{array}$ \\
\hline 16) Rhina armata & $\begin{array}{l}\text { Arica, Coquimbo, Puerto Montt a } \\
\text { Estrecho de Magallanes }\end{array}$ & $\begin{array}{l}\text { Compagno (1984a), } \\
\text { Meléndez et al. (1993), } \\
\text { Chirichigno \& Vélez (1998) }\end{array}$ \\
\hline 17) Triakis maculata & Arica, Playa Brava e Iquique & $\begin{array}{l}\text { Compagno (1984b), } \\
\text { Meléndez et al. (1993), } \\
\text { Chirichigno \& Vélez (1998) }\end{array}$ \\
\hline
\end{tabular}


taxonómicos, sistemáticos y geográficos de los tiburones, requisito fundamental para la investigación ictiológica y la correcta difusión científica y manejo pesquero, entre otras especializaciones científicas.

Como consecuencia de los nuevos registros de los taxa capturados en aguas chilenas se observó un aumento de 48 a 56 en el número de especies, sumando una familia previamente no registrada: Pseudocarcharhiniidae (Lamniformes) (Rubio et al. 2005, Meléndez et al. 2006, Reyes \& Hüne 2006). Al respecto, cabe señalar la confirmación de la presencia de Isurus paucus en la zona centro de Chile, captura efectuada el 2004 en Caldera y Paico, y cuya presencia fue también registrada en marzo del año 2007 en el Pacífico suroeste (32³0’S, 74³0’W) en Caleta Portales, Valparaíso (Bustamante et al. 2009), que junto con Sphyrna lewini (conocida en el Perú) no han sido confirmados en los registros de pesquerías artesanales e industrial, aumentando el rango de distribución de ambas especies (Sebastian et al. 2008).

Este aumento en el número de especies obedece a las nuevas y diversas exploraciones efectuadas en el mar chileno en los últimos tiempos, las que al ser cada vez más frecuentes, permitieron la captura y registro de nuevos ejemplares y por ende, enriquecer los conocimientos sobre la condrictiofauna chilena. No se descarta la posibilidad que en un futuro cercano el número siga aumentando.

La clave de tiburones presentada en este trabajo facilitará la diagnosis de las especies del Superorden Squalomorphi de Chile y su uso pretende ser una base y complemento para futuras investigaciones taxo-sistemáticas, de biodiversidad e ictiogeografía, en tiburones que habitan aguas chilenas, ya que se integraron todos los taxa del Superorden Squalomorphi de Chile, facilitando el estudio científico para evitar diagnosis erróneas e incompletas, con el aporte de datos tan importantes como la distribución geográfica de estos peces.

\section{Agradecimientos}

Los autores agradecen especialmente al Dr. Alexei M. Orlov (Principal Scientist, Russian Federal Research Institute of Fisheries and Oceanography, Moscow, Russia), por su colaboración en la traducción de artículos científicos desde el idioma ruso al inglés. Don León Matamala (Instituto de Zoología, Universidad Austral de Chile) por facilitar bibliografía. A la Dirección de Investigación y Desarrollo (DID) de la Universidad Austral de Chile, la cual a través de los Proyectos S-96-04 y S.2005-03, proporcionó información valiosa para esta contribución.

\section{LITERATURA CITADA}

Acuña E, JC Villarroel \& R Grau. 2002. Fauna íctica asociada a la pesquería del pez espada (Xiphias gladius Linnaeus). Gayana 66(2): 263-267.

Andrade I \& G Pequeño. 2008. Mesobathic chondrichthyes of the Juan Fernández seamounts: are they different from those of the central Chilean continental slope? Revista de Biologia Tropical 56(1): 181-190.

Arrizaga A. 1969-1970. Nuevos antecedentes para Centroscyllium granulosus Günther, 1880 (Pisces-Squalidae). Boletín de la Sociedad de Biología de Concepción 42: 221223.

Bahamonde N \& G Pequeño. 1975. Peces de Chile. Lista Sistemática. Publicación Ocasional, Museo Nacional de Historia Natural 21: 1-20.

Brito JL. 2004a. Hallazgo de Somniosus pacificus Bigelow \& Schroeder, 1944 (Squaliformes: Squalidae) en San Antonio, Chile central. Investigaciones Marinas 32(2): 137-139.

Brito JL. 2004b. Presencia del tiburón martillo Sphyrna zygaena (Carchariniformes: Sphyrnidae) y nuevo registro del tiburón espinudo Echinorhinus cookei (Squaliformes: Squalidae) en San Antonio, Chile central. Investigaciones Marinas 32(2): 141-144.

Bustamante C, F Concha, F Balbontín \& J Lamilla. 2009. First record of Isurus paucus Gitart Manday, 1966 (Elasmobranchii: Lamnidae) in the southeast Pacific Ocean with notes about migratory shark fishery. Revista de Biología Marina y Oceanografía 44(2): 523-526.

Compagno LJV. 1984a. FAO Species Catalogue. Part 1. Sharks of the world. An annotated and illustrated catalogue of sharks species known to date. Hexanchiformes to Lamniformes. FAO Fisheries Synopsis 125 4(1): 1-249.

Compagno LJV. 1984b. FAO Species Catalogue. Part 2. Sharks of the world. An annotated and illustrated catalogue of shark species known to date. Carcharhiniformes. FAO Fisheries Synopsis 125 4(2): 251-655.

Compagno LJV. 1988. Sharks of the order Carcharhiniformes, 486 pp. Princeton University Press, Nueva York.

Compagno LJV. 1999. Checklist of living elasmobranchs. En: Hamlett WC (ed). Sharks, skates and rays: the biology of elasmobranchs fishes, pp. 471-498. The Johns Hopkins University Press, Baltimore \& Londres.

Compagno LJV. 2001. Sharks of the world. An annotated and illustrated catalogue of shark species known to date. Volume 2. Bullhead, mackerel and carpet sharks (Heterodontiformes, Lamniformes and Orectolobiformes). FAO Species Catalogue for Fishery Purposes 1(2): 1-269.

Compagno LJV, M Dando \& S Fowler. 2005. Shark of the world, 368 pp. Princeton University Press, Princeton \& Oxford.

Cousseau MB \& RG Perrota. 2003. Peces marinos de Argentina. Biología, distribución, pesca, 41 pp. INIDEP, Mar del Plata.

Chirichigno FN. 1974. Clave para identificar los peces marinos del Perú. Informe, Instituto del Mar del Perú 44: 1-387. 
Chirichigno FN \& J Vélez. 1998. Clave para identificar los peces marinos del Perú, 302 pp. Publicación Especial, Instituto del Mar del Perú, Callao.

De Buen F. 1959. Lampreas, tiburones, rayas y peces de la Estación de Biología Marina de Montemar, Chile. Revista Biología Marina 9(1-3): 1-200.

Dolganov VN. 1984. A new shark from the family Squalidae caught on the Naska submarine ridge. Zoologicheskii Zhurnal 63(10): 1589-1591.

Eschmeyer B \& R Fricke. 2010. Catalog of fishes [en línea] $<$ http://research.calacademy.org/ichthyology/catalog >

Flores H \& P Rojas. 1979. Presencia de Echinorhinus cookei Pietschmann, 1928 frente a las costas de Chile (Pisces: Squalidae, Echinorhinidae). Investigaciones Marinas 7(1): 41-45.

Fowler HW. 1945. Fishes of Chile, Systematic catalog reprint. Apartado de la Revista Chilena de Historia Natural 45-47: 1-36, 1-171.

Fowler HW. 1951. Analysis of the fishes of Chile, Systematic Catalog. Revista Chilena de Historia Natural 51-53: 263326.

Garrick JAF. 1967. Revision of sharks of genus Isurus with description of a new species (Galeoidea, Lamnidae). Proceedings of the United States National Museum 118(3337): 663-690.

Garrick JAF. 1982. Sharks of the genus Carcharhinus. NOAA Technical Report, NMFS Circular 445: 1-194.

Gosztonyi AE. 1973. Sobre el dimorfismo sexual secundario en Halaelurus bivius (Müller y Henle, 1841) Garman 1913 (Elasmobranchii, Scyliorhinidae) en aguas PatagónicoFueguinas. Physis A 32(85): 317-323.

Gubanov YP \& VN Grigor'ev. 1975. Distribution and biology of the blue shark Prionace glauca (Carcharhinidae) of the Indian Ocean. Journal of Ichthyology 15: 37-43.

Guzmán L \& I Campodónico. 1976. Notorynchus cepedianus (Peron, 1807) en la entrada oriental del Estrecho de Magallanes (Elasmobranchii, Hexanchiidae). Anales del Instituto de la Patagonia 7: 207-210.

Hubbs CL. 1951. Record of Carcharhinus longimaus with Naucrates and Remora from the East-Central Pacific. Pacific Science 5: 78-81.

Iwasaki Y. 1970. On the distribution and environment of the whale shark, Rhincodon typus, in skipjack fishing grounds in the western Pacific Ocean. Journal of the College of Marine Science and Technology Tokai University 4: 37-51.

Kukuyev EI \& II Konovalenko. 1988. Two new species of sharks of the genus Scymnodalatias (Dalatiidae) from the North Atlantic and southeastern Pacific oceans. Voprosy Iktiologii 28(2): 315-319.

Lamilla J \& C Bustamante. 2005. Guía para el reconocimiento de: Tiburones, rayas y quimeras de Chile. Oceana 17: 1-80.

Long J. 1997. First account of the birdbeak dogfish shark, Deania calcea, (Chondrichthyes: Squalidae) from the northern coast of Perú. Revista de Biología Tropical 45(2): $1-937$.
Lloris D \& J Rucabado. 1991. Ictiofauna del canal Beagle (Tierra del Fuego), aspectos ecológicos y análisis biogeográfico. Publicación Especial, Instituto Español de Oceanografía 8: 1-182.

Mann G. 1954. La vida de los peces en aguas chilenas, 342 pp. Instituto de Investigaciones Veterinarias, Universidad de Chile, Santiago.

Meléndez R. 1991. Zameus squamulosus (Günther, 1877) en Chile. (Chondrichthyes, Squaliformes, Squalidae). Investigación Pesquera, Chile 36: 89-95.

Meléndez R \& D Meneses. 1986. Tiburones del talud continental entre Arica $\left(18^{\circ} 25^{\prime} \mathrm{S}\right)$ e Isla Mocha (38 $\left.15^{\circ} \mathrm{S}\right)$. Investigaciones Marinas 17: 3-73.

Meléndez R \& R Céspedes. 1996. Centrophorus squamosus (Bonnaterre, 1788) en aguas del Golfo de Ancud. Primer registro para Chile (Elasmobranchii: Squaliformes: Squalidae). Estudios Oceanológicos 15: 35-37.

Meléndez R, O Gálvez \& A Cornejo. 1993. Catálogo colección de peces depositada en el Museo Nacional de Historia Natural de Chile. Publicación Ocasional, Museo Nacional de Historia Natural 47: 1-224.

Meléndez R, S López \& E Yánez. 2006. Nuevos antecedentes de Pseudocarcharias kamoharai (Matsubara, 1936) (Chondrichthyes: Lamniformes: Pseudocarchariidae), frente al norte de Chile. Investigaciones Marinas 34(2): 223-226.

Menni RC \& LO Lucifora. 2007. Condrictios de la Argentina y Uruguay. ProBiota, FCNyM, UNLP. Serie Técnica y Didáctica 11: 1-14.

Moreno JA, JI Parajua \& J Morón. 1989. Biología reproductiva y fenología de Alopias vulpinus (Bonnaterre, 1788) (Lamniformes: Alopiidae) en el Atlántico nororiental y Mediterráneo occidental. Scientia Marina 53(1): 37-46.

Morillas J. 1977. Primer registro de Chlamydoselachus anguineus Garman frente a la costa de Chile. Noticiario Mensual, Museo Nacional de Historia Natural 21(250): 910.

Nakaya K \& AJ Bass. 1978. The frill shark, Chlamydoselachus anguineus in New Zealand seas. New Zealand Journal of Marine and Freshwater Research 12(4): 397-398.

Nelson JS. 1994. Fishes of the world, 600 pp. John Wiley \& Sons, New York.

Oñate J \& GP Pequeño. 2005. Etmopterus brachyurus Smith \& Radcliffe, 1912 (Chondrichthyes, Dalatiidae): primer registro en aguas del Pacífico oriental. Revista de Biología Marina y Oceanografía 40(1): 63-65.

Parin NV \& AN Kotlyar. 2007. On finding of shark of the genus Somniosus (Squalidae) at the submarine ridge of Nazca (southeastern Pacific). Journal of Ichthyology 47(8): 669672.

Pequeño G. 1977. El género Galeorhinus en Chile (Elasmobranchii: Triakidae). Revista de Biología Marina 16(2): 183-188.

Pequeño G. 1979a. El género Notorhynchus en Chile (Elasmobranchii). Revista de Biología Marina 16(3): $257-$ 264. 
Pequeño G. 1979b. Nota sobre un ejemplar de Cetorhinus maximus (Gunnerus, 1765) capturado frente a Corral, Chile. Neotropica 73: 97-98.

Pequeño G. 1981. Comentarios sobre Apristurus nasutus De Buen, 1959 (Elasmobranchii: Scyliorhinidae), en base a un nuevo registro. Boletín de la Sociedad de Biología de Concepción 52: 129-133.

Pequeño G. 1989. Peces de Chile. Lista sistemática revisada y comentada. Revista de Biología Marina 24: 1-132.

Pequeño G. 1997. Peces de Chile. Lista sistemática revisada y comentada: Addendum. Revista de Biología Marina y Oceanografía 32(2): 77-94.

Pequeño G \& S Sáez. 2003. Characteristics of Carcharhinus galapagensis from Salas y Gómez Island, Chile (Chondrichthyes: Carcharhinidae). Revista de Biología Tropical 51(3-4): 795-796.

Pequeño G \& S Sáez. 2004. Peces marinos comunes del litoral de Valdivia. Guía de reconocimiento para efectos prácticos, 87 pp. Ediciones Surambiente, Santiago.

Pequeño G, J Rucabado \& D Lloris. 1990. Tiburones comunes a las costas de Chile, California-Oregón y Namibia-Sudáfrica. Revista de Biología Marina 24(2): 65-80.

Pequeño G, J Lamilla \& A Crovetto. 1991. Captura de Somniosus cf. pacificus Bigelow y Schroeder, 1944, frente a Valdivia, Chile, con notas sobre su contenido gástrico (Chondrichthyes, Squalidae). Estudios Oceanológicos 10: 117-122.

Regan CT. 1908a. A synopsis of the sharks of the Family Scyliorhinidae. Annals and Magazine of Natural History, Serie 8 1(6): 453-465.

Regan CT. 1908b. A synopsis of the sharks of the Family Squalidae. Annals and Magazine of Natural History , Serie 8 2(7): 39-57.

Reyes P \& M Hüne. 2006. Primer registro del tiburón Etmopterus unicolor (Engelhardt, 1912) frente a Valdivia, Chile (Chondrichthyes: Dalatiidae). Investigaciones Marinas 34(2): 137-142.

Rios NH, C Ríos \& P Meneses. 2002. Peces del Uruguay: Lista sistemática y nombres comunes, 105 pp. INFOPESCA, Montevideo.

Rojas JR, C Fuentes \& NR Hernández. 2006. Echinorhinus cookei Pietschmann, 1928 (Chondrichthyes: Squaliformes, Echinorhinidae), primer registro en aguas de El Salvador, Pacífico oriental tropical. Revista de Biología Marina y Oceanografía 41(1): 117-119.

Rubio E, MJ Pedraza \& LA Zapata. 2005. Primer registro del tiburón perro Centroscyllium nigrum (Chondricthyes: Squalidae) en aguas del Pacífico colombiano. Gayana 29(1): 113-117.
Ruiz VH \& J Fernández. 1984. Primer registro de Echinorhinus cookei Pietschmann 1928 (Pisces, Squaliformes: Squalidae) para Chile. Boletín de la Sociedad de Biología de Concepción 55: 175-176.

Sadowsky V. 1973. First record of a basking shark Cetorhinus maximus (Gunnerus, 1765) in the Brazilian Atlantic. Boletim do Instituto Oceanográfico, Sao Paulo 22: 1-10.

Schaaf-Da Silva JA \& DA Ebert. 2008. A re-description of the eastern Pacific swellshark, Cephaloscyllium ventriosum (Garman 1880) (Chondrichthyes: Carcharhiniformes: Scyliorhinidae), with comments on the status of $C$. uter (Jordan \& Gilbert 1896). Zootaxa 1872: 59-68. [en línea] $<$ http://www.mapress.com/zootaxa/ 2008 /f/ z01872p068f.pdf>

Sebastian H, PA Haye \& MS Shivji. 2008. Characterization of the pelagic shark-fin trade in north-central Chile by genetic identification and trader surveys. Journal of Fish Biology 73: 2293-2304.

Siccardi EM. 1960. 'Cetorhinus' en el Atlántico sur (Elasmobranchii: Cetorhinidae). Revista del Museo Argentino de Ciencias Naturales Bernardino Rivadavia 6(2): 61-101.

Siccardi EM, AE Gosztonyi \& RC Menni. 1981. La presencia de Carcharodon carcharias e Isurus oxyrhyncus en el Mar Argentino (Chondrichthyes, Lamniformes). Physis A 39(97): 55-62.

Sielfeld W \& M Vargas. 1992. Tiburones y rayas del Pacífico Sur Oriental, 104 pp. Departamento de Ciencias del Mar, Universidad Arturo Prat, Iquique.

Smith JLB. 1957. Sharks of the genus Isurus Rafinesque, 1810. Ichthyological Bulletin of Rhodes University, Grahamstown 6: 91-96.

Stehmann M, EI Kukuyev \& II Konovalenko. 1999. Three new adult records of the oceanic longnose pygmy shark, Heteroscmynoides marleyi, from the Southeastern Atlantic and Southeastern Pacific (Chondrichthyes, Squaliformes, Squalidae). Journal of Ichthyology 39(8): 606-615.

Taniuchi $\mathbf{T}$ \& JAF Garrick. 1986. A new species of Scymnodalatias from the Southern Oceans, and comments on other Squaliform sharks. Japanese Journal of Ichthyology 33(2): 119-134.

Williams SF. 1970. Segundo registro para Chile del tiburón, Centrocymmus crepidater (Familia Squalidae). Investigaciones Marinas 1(8): 155-166.

Wolfson FH \& G Notarbartolo di Sciara. 1981. The whale shark, Rhiniodon typus Smith, 1828: an annotated bibliography (Selachii Rhiniodontidae). Atti dellaSocietà Italiana di Scienze Naturali e del Museo Civico di Storia Naturale di Milano 122(3-4): 171-203. 
Anexo 1. Lista sistemática de especies del Superorden Squalomorphi de Chile / Systematic list of species of Superorder Squalimorphi from

\section{HEXANCHIFORMES}

\section{HEXANCHIDAE}

Heptranchias perlo (Bonnaterre, 1788)

Hexanchus griseus (Bonnaterre, 1788)

Notorynchus cepedianus (Pèron, 1807)

\section{CHLAMYDOSELACHIDAE}

Chlamydoselachus anguineus Garman, 1884

\section{ORECTOLOBIFORMES}

\section{RHINCODONTIDAE}

Rhincodon typus Smith, 1828

\section{LAMNIFORMES}

\section{PSEUDOCARCHARIIDAE}

Pseudocarcharias kamoharai (Matsubara, 1936)

\section{LAMNIDAE}

Carcharodon carcharias (Linnaeus, 1758)

Isurus oxyrinchus Rafinesque, 1809

Isurus paucus Guitart Manday, 1966

Lamna nasus (Bonnaterre, 1788)

\section{CETORHINIDAE}

Cetorhinus maximus (Gunnerus, 1765)

\section{ALOPIIDAE}

Alopias superciliosus (Lowe, 1841)

Alopias vulpinus (Bonaterre, 1788)

\section{CARCHARHINIFORMES}

\section{SCYLIORHINIDAE}

Apristurus brunneus (Gilbert, 1892)

Apristurus nasutus De Buen, 1959

Bythaelurus canescens (Günther, 1878)

Cephaloscyllium ventriosum (Garman, 1880)

Cephalurus cephalus Gilbert, 1892

Schroederichthys bivius (Müller \& Henle, 1838)

Schroederichthys chilensis (Guichenot, 1848)

\section{TRIAKIDAE}

Galeorhinus galeus (Linnaeus, 1758)

Mustelus mento Cope, 1877

Mustelus whitneyi Chirichigno, 1973

Triakis maculata Kner \& Steindachner, 1867

\section{CARCHARHINIDAE}

Carcharhinus galapagensis (Snodgrass \& Heller, 1905)

Prionace glauca (Linnaeus, 1758)

\section{SPHYRNIDAE}

Sphyrna lewini (Griffith \& Smith, 1834)

Sphyrna zygaena (Linnaeus, 1758)

\section{SQUALIFORMES}

DALATIIDAE

Aculeola nigra De Buen, 1959

Centroscyllium granulatum Günther, 1887

Centroscyllium nigrum Garman, 1899

Centroscymnus crepidater (Barbosa du Bocage \& de Brito Capello, 1864)

Centroscymnus macracanthus Regan, 1906

Centroscymnus owstonii Garman, 1906

Etmopterus brachyurus Smith \& Radcliffe, 1912

Etmopterus granulosus (Günther, 1880)

Etmopterus litvinovi Parin \& Kotlyar, 1990

Etmopterus lucifer Jordan \& Snyder, 1902

Etmopterus pycnolepis Kotlyar, 1990

Etmopterus pusillus (Lowe, 1839)

Etmopterus unicolor (Engelhardt, 1912)

Etmopterus villosus Gilbert, 1905

Euprotomicrus bispinatus (Quoy \& Gaimard, 1824)

Heteroscymnoides marleyi Fowler, 1934

Isistius brasiliensis (Quoy \& Gaimard, 1824)

Mollisquama parini Dolganov, 1984

Scymnodalatias oligodon Kukuyev \& Konovalenko, 1988

Zameus squamulosus (Günther, 1877)

Somniosus pacificus Bigelow \& Schroeder, 1944

\section{ECHINORHINIDAE}

Echinorhinus cookei Pietschmann, 1928

\section{CENTROPHORIDAE}

Centrophorus squamosus (Bonnaterre, 1788)

Deania calcea (Lowe, 1839)

SQUALIDAE

Squalus acanthias Linnaeus, 1758

Squalus blainville (Risso, 1827)

Squalus mitsukurii Jordan \& Snyder, 1903

\section{SQUATINIFORMES}

SQUATINIDAE

Rhina armata Philippi, 1887 
Anexo 2. Glosario de términos utilizados en la clave / Glossary of terms used in the key

- $\quad$ Apex (=ápice). Extremo distal de una aleta.

- Barbos nasales. Proyecciones finas de consistencia carnosa o cartilaginosa, sensitivas situadas en las cercanías de la boca, mentón o región gular. Presentes en Squatina armata (Squatinidae).

- Cúspide. En el diente, proyección principal de la corona.

- Dentículos. Tipo de espinulación presente en la piel de tiburones y rayas. Son evidentes al tacto y poseen forma de estrella.

- Deprimido. Cuerpo aplastado dorsoventralmente, como en Squatinidae.

- Dientes molariformes (o en pavimento). Dientes aplanados, sin cúspides. Presentes por ejemplo en Mustelus mento (Triakidae).

- Dientes monocuspidiados, tricuspidiados. Dientes con una y tres cúspides respectivamente.

- Espiráculo. Reminiscencia de abertura branquial que se extiende desde la cavidad faríngea, desde la cual emerge, justo detrás del ojo.

- $\quad$ Falcada, aleta. En forma de hoz.

- Fosa precaudal. Hendidura que presentan algunos tiburones, situada en la línea media dorsal, o ventral del pedúnculo caudal. Presente en Carcharhinidae.

- Membrana nictitante. Membrana similar a los párpados, ubicada en el ángulo interno del ojo.

- Pliegues labiales. Formaciones carnosas situadas en los extremos de la abertura bucal en tiburones.

- Quilla (= quilla subcaudal). Parte saliente y a veces afilada, a lo largo y en los costados del pedúnculo caudal.

- Válvula espiral. Estructura intraintestinal en forma de tirabuzón. Su función es aumentar la capacidad de absorción de los alimentos. 